\title{
Flebótomos de áreas com ocorrências de casos humanos de leishmaniose tegumentar americana no Município de Seropédica, Estado do Rio de Janeiro
}

\author{
Sandflies in an areas with occurrences of human cases of American cutaneous \\ leishmaniasis in the municipality of Seropédica, State of Rio de Janeiro
}

\author{
Patrícia Giupponi Cardoso 1 , Marcos Barbosa de Souza², Argemiro Sanavria ${ }^{3}$, \\ Antonio de Medeiros Meira² e Jairo Caetano Meródio ${ }^{2}$
}

\begin{abstract}
RESUMO
A leishmaniose tegumentar americana é causada por protozoários do gênero Leishmania, que acomete a pele e mucosa; é primariamente uma infecção zoonótica. Inquéritos flebotomínicos foram realizados em quatro áreas com ocorrência de casos humanos de leishmaniose tegumentar americana do município de Seropédica. Utilizou-se a metodologia de coleta com capturas manuais no horário das 18 às $22 \mathrm{~h}$, durante 12 meses consecutivos. No período de outubro de 2004 e setembro de 2005, capturamos 2.390 exemplares pertencentes a quatro espécies, Lutzomyia intermedia com 97,1\% do total coletado, seguida pela Lutzomyia whitmani 1,6\%, Lutzomyia migonei 1,2\% e Lutzomyia oswaldoi $0,1 \%$. 0 Valão da Louça apresentou maior densidade flebotomínica, seguida pelas localidades de Km 39, Km 40 e Caçador. A baixa diversidade de espécie se deve a alterações significativas do ecótopo da região. Sugere-se que a transmissão da leishmaniose tegumentar americana no município de Seropédica esteja sendo veiculada pela Lutzomyia intermedia.
\end{abstract}

Palavras-chaves: Leishmaniose tegumentar americana. Flebotomíneos. Lutzomyia.

\begin{abstract}
American cutaneous leishmaniasis is caused by protozoa of the genus Leishmania, which affects the skin and mucosa. It is primarily a zoonotic infection. Sandfly surveys were conducted in four areas with occurrences of human cases of American cutaneous leishmaniasis in the municipality of Seropédica. A manual method was used to catch specimens between 6:00 pm and 10:00 pm, for 12 consecutive months. From 0ctober 2004 to September 2005, we caught 2,390 individuals belonging to four species: Lutzomyia intermedia accounted for $97.1 \%$ of the total collected, followed by Lutzomyia whitmani (1.6\%), Lutzomyia migonei (1.2\%) and Lutzomyia oswaldoi $(0.1 \%)$. The Valão das Louças had the highest density of sandflies, followed by the localities of Km 39, Km 40 and Caçador. The low diversity of species is due to significant changes in the ecotope of the region. It is suggested that American cutaneous leishmaniasis is being transmitted by Lutzomyia intermedia in the municipality of Seropédica.
\end{abstract}

Key-words: American cutaneous leishmaniasis. Sandflies. Lutzomyia.

A leishmaniose tegumentar americana (ITA) apresenta-se amplamente no continente americano, ocorrendo desde o sul dos Estados Unidos ao norte da Argentina. Apesar da sua endemicidade em várias regiões do continente Sul Americano, sua real prevalência não é muito bem estabelecida, em função dos sub-registros de dados; diagnósticos diferenciais existentes; infecções inaparentes; variações de resposta do hospedeiro e de agentes etiológicos envolvidos ${ }^{45}$.

A implicação dos flebotomíneos como vetor da leishmaniose foi demonstrado, experimentalmente ${ }^{2}$, no antigo Estado da

\footnotetext{
1. Curso de Pós-graduação em Ciências Veterinárias, Universidade Federal Rural do Rio de Janeiro, Rio de Janeiro, RJ. 2. Departamento de Ciências Biológicas, Escola Nacional de Saúde Pública, Fundação Oswaldo Cruz, Rio de Janeiro, RJ. 3. Departamento de Epidemiologia e Saúde Pública, Instituto de Veterinária, Universidade Federal Rural do Rio de Janeiro, Rio de Janeiro, RJ. Endereço para correspondência: Dra. Patrícia Giupponi Cardoso. Rua Maia de Lacerda 91/105, Estácio, 20250-001 Rio de Janeiro, RJ,

Tel: $55219337-4571$

e-mail: pgcvet@gmail.com

Recebido para publicação em 23/06/2007

Aceito em 11/02/2009
}

Guanabara, capital do Rio de Janeiro, ao inocular e reproduzir a lesão no focinho de um cão a partir de macerado do conteúdo intestinal de Lutzomyia intermedia.

Na década de 50, foi registrado um surto de leishmaniose tegumentar americana no interior do Estado do Rio de Janeiro, mais precisamente no município de Magé, cujas aspersões de diclorodifeniltricloroetano (DDT) utilizada na campanha de combate ao mosquito da malária, que também afetavam os flebotomíneos $^{18}$, reduziram consideravelmente 0 número de casos desta protozoose.

Com a suspensão do programa da malária e conseqüentemente a suspensão das dedetizações na década de 70 ocorreu um recrudescimento da LTA na maioria das regiões brasileiras ${ }^{11}$. Deste modo, passou a ser registrada em baixas incidências com surtos epidêmicos esporádicos, em áreas de colonização antiga e onde se verificavam ocupações humanas desordenadas ${ }^{15} 24$.

Atualmente, a LTA é endêmica em vários municípios do Estado do Rio de Janeiro, atingindo pessoas de diferentes faixas 
etárias, caracterizando a transmissão intra e peridomiciliar. A Lutzomyia intermedia, pré-adaptada ao ambiente alterado, passou a ser considerado vetor potencial desta protozoose $\mathrm{e}^{1922}$ acompanhada, mais recentemente no município do Rio de Janeiro pela Lutzomyia migone $i^{19}$.

Com a municipalização dos programas de leishmanioses, o município de Seropédica notificou no ano de 2003, quinze casos humanos autóctones de LTA, sendo que, nove casos foram procedentes da localidade de Valão da Louça. Embora, houvesse registros de ocorrências de casos autóctones de LTA na supracitada localidade, pode-se constatar que havia escassez de estudos sistemáticos envolvendo vetores das leishmanioses.

\section{MATERIAL E MÉTODOS}

O Município de Seropédica é um dos mais recentes do estado do Rio de Janeiro, criado através da Lei Estadual 2.446 em 12/10/1995; emancipado do município de Itaguaí. As coordenadas geográficas correspondem a $22^{\circ}$, $45^{\prime}$ de latitude Sul e a $43^{\circ}, 41^{\prime}$ de longitude Oeste. Seropédica está localizada na região Oeste do Estado supracitado, Baixada Fluminense, distante 70 quilômetros da capital. A extensão territorial é de $274 \mathrm{Km}^{2}$, possuindo 33 metros de altitude acima do nível do mar. A população foi estimada em 65.020 habitantes. As atividades econômicas estão concentradas no comércio, extração mineral, indústria, atividades agrícolas (lavoura de banana) e a pecuária.

O estudo da fauna flebotomínica foi realizado no Município de Seropédica, Estado do Rio de Janeiro, em áreas com notificações de casos humanos e caninos de leishmaniose tegumentar americana. As localidades com casos índices de LTA selecionadas foram: Valão da Louça e Caçador, áreas predominantemente rurais e KM 39 e Km 40, áreas peri-urbanas. Para avaliar a presença de flebotomíneos e a possível transmissão da LTA foram realizadas coletas sistemáticas deste díptero, com o intuito de avaliar a frequiência sazonal dos vetores, e possíveis infecções naturais por Leishmania sp. A princípio estavam estabelecidas coletas no intradomicílio, entretanto, houve rejeição dos moradores em face da violência na região. Foram realizadas coletas sistemáticas mensais no período de outubro de 2004 a setembro de 2005 nos peridomicílios, paredes externas dos domicílios e abrigos de animais domésticos. Nas áreas selecionadas os animais domésticos não apresentavam abrigos fixos como galinheiros e canil, apenas um curral que esporadicamente um eqüino se fazia presente. Deste modo, as coletas foram direcionadas para os locais onde os animais se situavam no dia de cada coleta.

As coletas com o tubo capturador de Castro ${ }^{7}$ foram efetuadas no horário das $18 \mathrm{~h}$ às $22 \mathrm{~h}$ ( $4 \mathrm{~h}$ de captura/área). Foram empregadas fichas de campo para anotações referentes às capturas, como as temperaturas, umidade relativa do ar, ventos e chuvas. Os flebótomos coletados foram introduzidos em frascos contendo álcool $70^{\circ} \mathrm{e}$ transportados para o laboratório. Durante a clarificação os insetos são colocados em pequenas placas de Petri, numa solução de hidróxido de potassa (KOHI a 10\%, onde permanecem entre 2-3 horas, para que haja o amolecimento da quitina; após esse período são transferidos para outras placas de Petri com ácido acético, por um período de 15-20 minutos, para retirar o excesso de hidróxido de potassa; a seguir, lava-se em água destilada por 20 minutos; os insetos permanecem em lactofenol por 24 horas, para diafanizar; em seguida são montados entre lâmina e lamínula, em líquido de Berlese, e as lâminas colocadas para secar horizontalmente).

Após realizar os procedimentos de preparação e montagem, em ambas as técnicas, as lâminas montadas são levadas ao microscópio bacteriológico para identificação de acordo com a nomenclatura proposta por Young \& Duncan ${ }^{28}$.

Foram utilizados 150 flebotomíneos previamente dissecados e identificados como Lutzomyia intermedia, totalizando 15 pools com 10 exemplares fêmeas. Cada exemplar foi dissecado com auxilio de agulhas descartáveis após romper o penúltimo segmento e observar as espermatecas. Os machos foram identificados diretamente através do exame das genitálias com o auxilio de estereomicroscópio (40x). Deve-se ressaltar que para cada fêmea dissecada foram utilizadas apenas duas agulhas descartáveis, evitando assim a contaminação do material a ser utilizado nas provas de reação em cadeia de polimerase (PCR).

0 ácido desoxirribonucléico total usado na PCR é obtido do pool de amostras contendo 10 abdomens de flebótomos em tampão de lise $(\mathrm{pH} \mathrm{8,0)}$ contendo $100 \mu \mathrm{g} / \mathrm{ml}$ de proteinase $\mathrm{K}$ (Invitrogen Life Tecnologies, Carlsbad, CA, USA). As preparações foram mantidas em tubos eppendorf e incubadas $\left(2\right.$ horas, $\left.56^{\circ} \mathrm{C}\right)$ com agitações periódicas. Ao final da incubação o material foi centrifugado (13.000 rpm; $4^{\circ} \mathrm{C} ; 15$ minutos) e o sobrenadante coletado e aquecido $\left(95^{\circ} \mathrm{C}, 15\right.$ minutos) para inativar a proteinase K. O material resultante da precipitação foi submetido ao fenol clorofórmio. Posteriormente, as amostras foram mais uma vez centrifugadas para ressuspender o DNA em $50 \mu \mathrm{g} / \mathrm{ml}$ de tampão lise (pH 8,0). Uma porção de 10 microlitros do DNA extraído foi usada para amplificação. Procedimentos rigorosos foram adotados para reduzir a possibilidade de contaminação, por exemplo, a inclusão de grupos de machos de flebotomíneos como controle negativo, na extração do DNA e a descontaminação dos instrumentos e trabalhar em áreas descontaminadas com solução de cloreto e raios UV.

0 ácido desoxirribonucleico total foi obtido de culturas de promastigotas de Leishmania (Viannia) braziliensis usado como controle positivo. Parasitos foram colhidos em fase logarítmica elavados em PBS antes do isolamento do DNA. Depois solução tampão de lise contendo fenol clorofórmio e precipitação do etanol, como descritas acima para amostras dos insetos de acordo com Pita-Pereira ${ }^{19}$.

\section{RESULTADOS}

Durante o período de outubro de 2004 a setembro de 2005, foram coletados 2.390 flebótomos pertencentes a quatro espécies: Lutzomyia (Nyssomyia) intermedia (Lutz \& Neiva 1912). Lutzomyia migonei (França, 1920). Lutzomyia (Nyssomyia) whitmani (Antunes \& Coutinho, 1939). Lutzomyia oswaldo (Mangabeira, 1940). 
Como em todas as áreas endêmicas de leishmaniose tegumentar americana a espécie predominante foi a Lutzomyia intermedia com 2.120 (91,3\%) machos e 202 (8,7\%) fêmeas. A Lutzomyia whitmani foi a segunda espécie mais freqüente com $35(94,5 \%)$ machos e 2 (5,5\%) fêmeas, seguida pela Lutzomyia migonei com $20(68,9 \%)$ machos e $9(31,1 \%)$ fêmeas e Lutzomyia oswaldoi com apenas 2 (100\%) fêmeas (Tabela 1).

\section{TABELA 1}

Número e percentuais de flebótomos coletados no município de Seropédica, RJ, período: outubro de 2004 a setembro de 2005.

\begin{tabular}{|c|c|c|c|c|c|c|}
\hline \multirow[b]{2}{*}{ Espécies } & \multicolumn{2}{|c|}{ Machos } & \multicolumn{2}{|c|}{ Fêmeas } & \multicolumn{2}{|c|}{ Total } \\
\hline & $\mathrm{n}^{0}$ & $\%$ & $\mathrm{n}^{\underline{0}}$ & $\%$ & $\mathrm{n}^{0}$ & $\%$ \\
\hline Lutzomyia intermedia & 2.120 & 97,5 & 202 & 93,9 & 2.322 & 97,1 \\
\hline Lutzomyia whitmani & 35 & 1,6 & 2 & 0,9 & 37 & 1,6 \\
\hline Lutzomyia migonei & 20 & 0,9 & 9 & 4,3 & 29 & 1,2 \\
\hline Lutzomyia oswaldo $i$ & 0 & 0,0 & 2 & 0,9 & 2 & 0,1 \\
\hline Total & 2.175 & 100,0 & 215 & 100,0 & 2.390 & 100,0 \\
\hline
\end{tabular}

Quanto ao percentual total de flebótomos, capturados por áreas trabalhadas, obteve-se os seguintes resultados: Valão da Louça: 36,5\%, Km 39: 29,8\%, Km 40: 22,2\% e Caçador: 11,5\% (Figura 1).

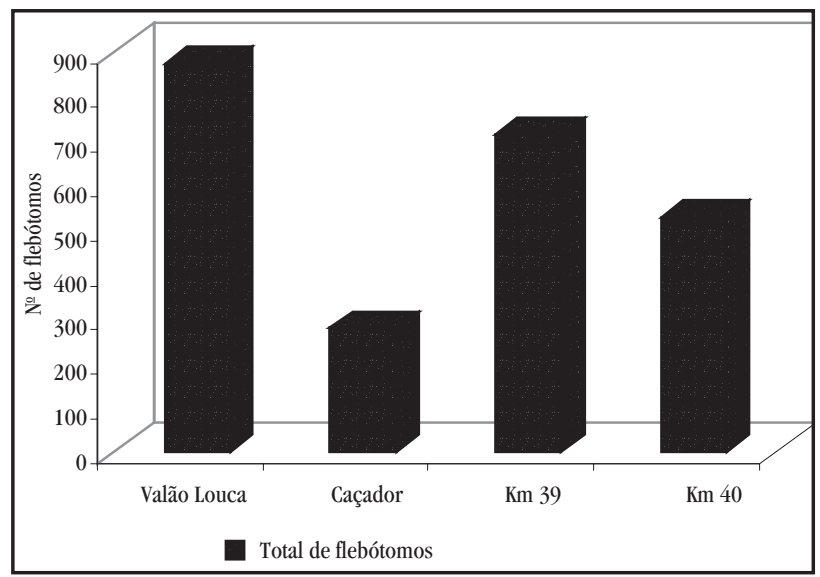

FIGURA 1

Total de flebótomos coletados nas quatro áreas com ocorrência de leishmaniose tegumentar americana no Município de Seropédica, RJ, no período de outubro de 2004 a setembro de 2005 .

No computo geral, os meses de maiores densidades flebotomínica foram verificados em outubro de 2004 e julho de 2005, cujas densidades foram 463 e 508 exemplares respectivamente. Deve-se ressaltar que a baixa incidência de flebótomos nos meses de janeiro e maio de 2004 ocorreu em função das fortes precipitações pluviométricas que impediram a realização das coletas (Figura 2). A relação, número de machos e fêmeas, capturados em todas as áreas trabalhadas foi muito grande, 2.175 machos contra apenas 215 fêmeas, ou seja, uma razão total de macho/fêmeas de aproximadamente 9:1 (Figura 3).

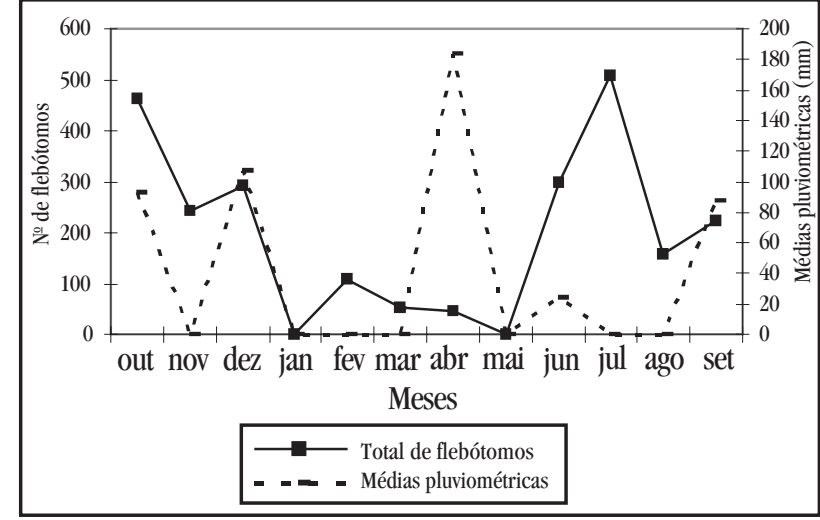

FIGURA 2

Frequência de flebótomos de acordo com as medidas das precipitações pluviométricas no Município de Seropédica, RJ, no período de outubro de 2004 a setembro de 2005.

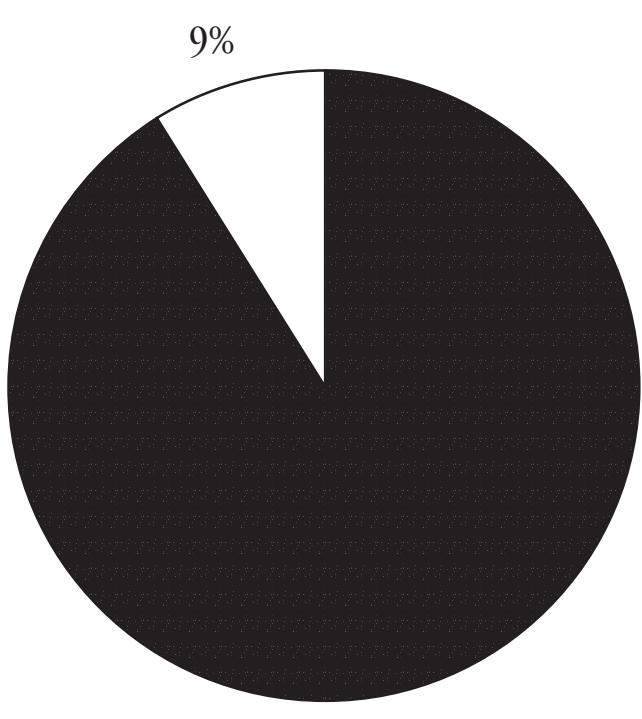

$91 \%$

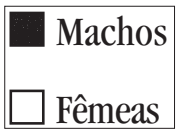

\section{FIGURA 3}

Relação entre números totais de machos e fêmeas de flebótomos coletados no Município de Seropédica, RJ no período de outubro de 2004 a setembro de 2005.

\section{DISCUSSÃO}

A fauna flebotomínica das áreas autóctones de Leishmaniose tegumentar americana no Município de Seropédica apresentou baixa diversidade de espécies, ou seja, apenas quatro espécies, não corroborando com os resultados obtidos no município de Seropédica ${ }^{26}$ que apresentou sete espécies. A baixa diversidade de espécies de flebótomos neste trabalho se deve provavelmente as coletas realizadas, exclusivamente, nos peridomicílios. Entretanto, 
deve ser ressaltado que a região apresenta características paisagísticas plana, cuja topografia apresenta 69\% de área de pasto contra $7 \%$ da sua vegetação secundária.

0 principal vetor de Leishmania (Viannia) braziliensis no Rio de Janeiro vem sendo a Lutzomyia intermedia. No bairro de Cosme Velho, Rio de Janeiro, Aragão $0^{2}$ reproduziu lesões no focinho de um cão ao inocular um pool de fêmeas de Lutzomyia intermedia. No bairro de Vargem Grande, Jacarepaguáa ${ }^{11}$ diagnosticou a infecção natural desta mesma espécie por Leishmania. Mais recentemente ${ }^{19}$ registrou Lutzomyia intermedia e Lutzomyia migonei com infecção natural em diferentes bairros do município do Rio de Janeiro.

A Lutzomyia whitmani também está associada à transmissão de Leishmania braziliensis, porém, nos estados da Bahia e Minas Gerais $^{1627}$ a principal caracterização de Lutzomyia migonei na participação da cadeia epidemiológica da LTA em área urbana foi mais bem evidenciada no Estado do Ceará, cujo vetor em área silvestre é a Lutzomyia wellcome ${ }^{23}$.

A espécie Lutzomyia intermedia prevaleceu em todos os locais de captura, confirmando a sua grande valência ecológica em ambientes alterados ${ }^{12}{ }^{14}$. Diferentemente das outras áreas endêmicas do Estado do Rio de Janeiro, a Lutzomyia whitmani foi à segunda espécie mais freqüente, substituindo a Lutzomyia migonei que comumente precede a Lutzomyia intermedia como a segunda espécie mais freqüente nas áreas endêmicas de ITA ${ }^{6}$. Apesar da baixa densidade de Lutzomyia whitmani, é importante observar que esta espécie é tida como o principal vetor de Leishmania (Viannia) braziliensis em diversas regiões do país ${ }^{1620}$ e associado à transmissão de Leishmania (Viannia) guyanensis e Leishmania (Viannia) shawi ${ }^{13}$.

A razão total de macho/fêmeas próximo de 9:1 caracteriza que as coletas manuais foram realizadas muito próximas dos criadouros, e é sabido que os machos nascem primeiro que as fêmeas e os mesmos se agrupam com o objetivo de acompanhar as fêmeas para o acasalamento ${ }^{8}$.

$\mathrm{Na}$ região sudeste, diversos autores verificaram baixa densidade de Lutzomyia intermedia nos meses mais frios do ano (junho e julho) ${ }^{3910}$. No município de Itaguaí ${ }^{1}$, obtiveram os menores índices de densidade de Lutzomyia intermedia em julho, agosto e setembro. No município de São José do vale do Rio Preto, verificaram que a espécie Lutzomyia intermedia apresentava maior pico de atividade no mês de junho ${ }^{25}$. Estes dados não corroboram com os resultados obtidos no município de Seropédica, cujo pico foi obtido no mês de julho. Nos diferentes trabalhos, relacionados à frequiência mensal de Lutzomyia intermedia, existem nítidas discrepâncias de resultados, tal fato ocorre, provavelmente, em função das diferentes características fisio e fito geográfico de cada região e a metodologia de coleta realizada em cada trabalho.

A taxa de infecção natural dos flebotomíneos, utilizando PCR, encontrada neste estudo, foi nula. Em uma área endêmica de LTA na Bahia, foi obtido um índice de 0,4\%, taxa suficiente para a manutenção da endemicidade da infecção ${ }^{17}$. A investigação da infecção em flebotomíneos capturados de forma não direcionada mostra uma taxa ainda mais baixa ou mesmo nula ${ }^{27}$.
Dentre as espécies capturadas neste estudo, desconsiderando a densidade das mesmas nas localidades de Seropédica, Lutzomyia intermedia, Lutzomyia migonei e Lutzomyia whitmani são reconhecidamente vetores de LTA no Brasil, sugere-se que as espécies Lutzomyia intermedia, Lutzomyia migonei e Lutzomyia whitmani sejam possíveis vetores da LTA no município de Seropédica.

\section{REFERÊNCIAS}

1. Aguiar GM, Vilela ML, Lima RB. Ecology of the sandflies of Itaguaí, na area of cutaneous leishmaniasis in the State of Rio de Janeiro. Food preferences (Diptera, Psychodidae, Phlebotominae). Memórias do Instituto Oswaldo Cruz 82:583-584, 1987.

2. Aragão HB. Transmissão da leishmaniose no Brasil pelo Pblebotomus intermedius. Brazil Médico 36:129, 1922.

3. Araújo-Filho NA. Epidemiologia da Leishmaniose Tegumentar Americana na Ilha Grande, Rio de Janeiro, Estudo sobre a infecção humana, reservatórios e transmissores. Dissertação de Mestrado, Universidade Federal Rural do Rio de Janeiro, Itaguaí, RJ, 1978.

4. Grimaldi Jr G, Tesh RB, MacMahon Pratt D. A review of the geographical distribution and epidemiology of leishmaniasis in the New World. The American Journal of Tropical Medicine and Hygiene 41:687-725, 1989.

5. Herrer A, Christensen HA. Epidemiological patterns of cutaneous leishmaniasis in Panama. III. Endemic persistence of the disease. The American Journal of Tropical Medicine and Hygiene, 25:54-58, 1976.

6. Carvalho RW, Serra-Freire NM, Souza MB. Fauna de flebótomos da Ilha do Araújo, município de Paraty-RJ. 1 - Diversidade e aspecto do comportamento. Parasitology Al Dia 19:104-112, 1995.

7. Castro G0. Sobre um processo de cultura de flebótomos. Nota prévia. In: Sociedade de Biologia, sessão de 8 de outubro, Rio de Janeiro, 1937.

8. Feliciangeli MD. Ecology of sandflies (Diptera: Psychodidae) in a restricted focus of cutaneous leishmaniasis in northern Venezuela, I. Description of the study area, catching methods and species composition. Memórias do Instituto Oswaldo Cruz 82: 119-124, 1987.

9. Forattini OP. Algumas observações sobre biologia de flebótomos (Diptera, Psychodidae) em região da bacia do Rio Paraná. Arquivo da Faculdade de Higiene e Saúde Pública, Universidade de São Paulo 8:40-86, 1954.

10. Forattini OP. Sobre os reservatórios naturais da leishmaniose tegumentar americana. Revista do Instituto de Medicina Tropical 2:195-203, 1960.

11. Fundação Nacional de Saúde. Leishmaniose Tegumentar Americana: Vigilância e Monitoramento da Leishmaniose Tegumentar em Unidades Territoriais-Brasil, 1994-2001. Boletim (eletrônico) Epidemiológico, Ano 02, № 05, 13/12/2002. www. funasa. gov.br, Ministério da Saúde, Brasília, 2002.

12. Gomes AC, Rabello EX, Santos JL, Galati EA. Aspectos ecológicos da leishmaniose tegumentar americana, 1. Estudo experimental da frequiência de flebotomíneo e ecótopos artificiais com referência especial a Psychodopygus intermedius. Revista de Saúde Pública 14:540-556, 1980.

13. Lainson R, Braga RR, Souza AAA, Póvoa MM, Ishikawa EAY, Silveira FT. Leishmania (Viannia) shawi sp.n. a parasite of monkeys, sloths and procyonids in Amazonian Brazil. Annales Parasitologie Humanaine et Comparee 64:200-207, 1989.

14. Lima LC. Ruralização da Lutzomyia intermedia, um provável caso de préadaptação. Revista de Saúde Pública 22:102-104, 1986.

15. Lima LCR, Marzochi MCA, Sabroza PC, Souza MA. Observações sobre leishmaniose tegumentar cinco anos após profilaxia. Revista de Saúde Pública 22:73-77, 1988.

16. Mayrink W, Williams P, Coelho MV, Martins AV, Magalhães PA, Costa CA, Falcão AL. Epidemiology of dermal leishmaniasis in the rio Doce valley, state of Minas Gerais, Brazil. Annais Tropical Medicine Parasitology 73:123-137, 1979.

17. Miranda JC, Reis E, Schriefer A, Gonçalves M, Reis MG, Carvalho L, Fernandes 0, Barral-Neto M, Barral A. Frequency of infection of Lutzomyia phlebotomines with Leishmania braziliensis in a Brazilian endemic área as 
assessed by pinpoint capture and polymerase chain reaction. Memórias do Instituto Oswaldo Cruz 97: 185-188, 2002.

18. Nery-Guimarães F. Estudo de um foco de leishmaniose mucocutânea na Baixada Fluminense (Estado do Rio de Janeiro). Memórias do Instituto Oswaldo Cruz 53:1-11, 1955.

19. Pita-Pereira D, Alves CR, Souza MB, Brazil RP, Bertho AL, Barbosa AF, Britto CC. Identification of naturally infected Lutzomyia migone $i$ with Leishmania (Viannia) braziliensis in Rio de Janeiro (Brazil) revealed by a PCR multiplex non-isotopic hybridisation assay. Transaction of the Royal Society of Tropical Medicine and Hygiene 99:905-913, 2005.

20. Queiroz RG, Vasconcelos AW, Vasconcelos A, Sousa RN, Pessoa FA, Alencar JE, David JR. Phlebotominae sand flie (Diptera: Psychodidae) fauna survey in na American cutaneous leishmaniasis (ACL) focus in Baturité, Ceará State, northeast Brazil. Parassitologia 33:159-167, 1991.

21. Rangel E, Ryan L, Lainson R, Shaw JJ. Observation on the sandfly (Diptera, Psychodidae) fauna of Alem Paraiba, state of Minas Gerais, Brazil, and the isolation of a parasite of the Leishmania braziliensis complex from Psychodopygus birsuta hirsuta. Memórias do Instituto Oswaldo Cruz 80: 373-374, 1985.

22. Rangel EF, Souza NA, Wermelinger ED, Barbosa AF. Infecção natural de Lutzomyia intermedia Lutz \& Neiva 1912, em área endêmica de leishmaniose tegumentar no Estado do Rio de Janeiro. Memórias do Instituto Oswaldo Cruz 79:395-396, 1984.
23. Ready PD, Ribeiro AL. Lainson R, Alentar JE, Shaw JJ. Presence of Psychodopygus wellcomei (Diptera, Psychodidae) a proven vector of Leishmania braziliensis in Ceará State. Memórias do Instituto Oswaldo Cruz 78:235-236, 1983.

24. Sabroza PC. O domicílio como fator de risco na leishmaniose tegumentar americana. Estudo epidemiológico em Jacarepaguá, município do Rio de Janeiro. Tese de Doutorado, Escola Nacional de Saúde Pública, Fundação Oswaldo Cruz, Rio de Janeiro, 1981.

25. Souza MB, Marzochi MCA, Carvalho RW, Conceição NF, Ponte CS. Flebotomíneos em áreas de ocorrência de leishmaniose tegumentar no município de São José do Vale do Rio Preto, Rio de Janeiro, Brasil. Parasitologia Al Dia 19:97-103,1995.

26. Souza MB, Péche WES, Borja GEM. Fauna Flebotomínica do Município de Seropédica, Rio de Janeiro: Área de Ocorrência de Leishmaniose Tegumentar Humana e Canina. In: XVIII Congresso Brasileiro de Parasitologia, Rio de Janeiro, p. $120,2003$.

27. Vexenat JA, Barretto AC, Cuba CC, Marsden PD. Epidemiological characteristics of American cutaneous leishmaniasis in an endemic region of the State of Bahia. III. Phlebotomine fauna. Memórias do Instituto Oswaldo Cruz 81: 293-301, 1986.

28. Young DG, Duncan MA. Guide to identification and geographic distribution of Lutzomyia sandflies im México, West Indies, Central and South America (Diptera: Psychodidae). Associated Publishers, American Entomological Institute, 1994. 